\title{
Application of Combination of High- Dose Amoxicillin with Nano-Silver and $70 \%$ Ethanol for the Treatment of a Recurrent Middle Ear Infection: A Case Report
}

\author{
Hasan Hosainzadegan ${ }^{1}$ \\ ${ }^{I}$ Department of Basic Sciences, Faculty of Medicine, Maragheh University of Medical Sciences, \\ Maragheh, Iran.
}

*Corresponding Author: Hasan Hosainzadegan, Department of Basic Sciences, Faculty of Medicine, Maragheh University of Medical Sciences, Maragheh, Iran.

\begin{abstract}
Acute otitis media (AOM) is a common childhood disease. Treatment of AOM cases has many problems for both patients and physicians, and in some cases recurrences of disease complicates the patient's condition. The aim of this case was to report the treatment of a recurrent AOM case with sequential treatment failures. The patient had a history of 2- year complicated form of chronic and acute otitis media with apparent criteria of approved AOM case. After 5 times of treatment failures by first line antibiotics, a combination of high-dose amoxicillin, silver nanoparticles, and ethanol $70 \%$ was prescribed for 7-day. After the mentioned treatment method, clinical condition, and audiometric parameters of patient changed to normal results. Although it seems like immoral, but considering one year follow-up after recovery without AOM recurrency and any other complications, this treatment regime is proposed for further clinical trials by ENTs.
\end{abstract}

Keywords: Otitis media, Recurrent, Nanoparticle, Treatment failure.

\section{INTRODUCTION}

Chronic and acute otitis media (AOM) are usually from common childhood infections that encountering with treatment failures. The empirical treatments, lack of reference laboratories for definite identification of etiology, and epidemiology of microbial infections, and indetermination of precise pattern of drug resistance in strains native to each region are among the causes of the treatment failures. Streptococcus pneumonia is one of the most important causes of antibiotic resistant organisms in AOMs resulting to treatment failures, after that organisms including S.aureus, H.influenzae, hemolytic and pyogenic streptococci are major causes of treatment failures in AOM. (1, 2) Unfortunately in failures there aren't more reliable options to treatment of recurrent AOMs, especially when Streptococcus pneumonia and H.influenzae are causative agents (3). Short course antibiotics (less than 7 days) are from other causes of treatment failures. (4). According to the descriptions, and because of the probability of the presence of resistant bacterial pathogens or viruses as etiology of the complicated conditions in an 5 year child with repeated treatment failures of AOM, using high doses of amoxicillin with nanosilver, and $70 \%$ ethanol have been proposed and applied for patient treatment.

\section{CASE Presentation}

The patient's is a 5-year-old male with a history of intermittent middle ear infections that last for 2 years of sever earaches and fever. Attacks of infection were repeated more than 5 times annually. According to the guidelines of the Centers for Disease Control and prevention (CDC) and American ENTs association this case could be considered as a known case of acute otitis media (AOM) and an indexed chronic middle ear infection.

Sudden attacks of severe earaches, fever, and vomiting were chief complaints of the patients, which majority of episodes occurring at nights. Additionally upper respiratory tract complications including plenty of sputum, dyspnea, and snoring were also complaints of the patients. Tympanometry and audiometry of both ears have indicated internal pressures and some degree of hearing loss. Otoscopy indicated the bulging of eardrum. In 3 of the recurrences the patient has been awakened from night 
sleep by earaches and crying, and 5 different episodes of disease treatment have been encountered with failures. The 6 treatment regimes and outcomes have been indicated in (Table.1).

Table1. Treatment regimes which has been prescribed for treatment of different episodes of AOM recurrences

\begin{tabular}{|c|c|c|}
\hline Variables & Treatment Regime & Result \\
\hline First Episode & Amoxicillin(50 Mg/Kg/Day)+Acetaminophen & Mild recovery \\
\hline Second Episode & IM Ceftriaxone+ Acetaminophen & Mild recovery \\
\hline Third Episode & IM Ceftriaxone+ Acetaminophen & Mild recovery \\
\hline Forth Episode & Cefixime (syrup) & Mild recovery \\
\hline Fifth Episode & Amoxicillin(85Mg/Kg/Day)+Acetaminophen+ Decongestant & Mild recovery \\
\hline Sixth Episode & Amoxicillin(85Mg/Kg/Day)+20ppm Nanosilver70\% Ethanol & Complete recovery \\
\hline
\end{tabular}

At $4^{\text {th }}$ episode of disease in spite of the complications of the cefixime on digestive tract, treatment course have been completed by patient. But the patient has been referred to the clinic with same signs and symptoms shortly after completing the treatment. As stated earlier in all of the recurrences of the disease a slight and periodic recovery of signs and symptoms have been occurred after treatments. Because of 5 times of treatment failures, combinations' of high-dose amoxicillin $(85 \mathrm{Mg} / \mathrm{Kg} / \mathrm{Day})$, 20ppm Nanosilver and 70\%Ethanol have been given to the patient. Adding unapproved agents for a treatment regimen maybe seems immoral, but has been proposed and conducted by complete consent of the patients parents especially because of severe earaches in the right ear of patient. Result of this treatment regimen was very satisfying for patient, because the audiometry of both ears after treatment was very attractive and indicates recovery of ears pressure to its normal ranges as indicated in figures 1 and 2. Nanosilver and 70\%ethanol was poured only one time to patient's ears. Based on 3 different ENTs examinations and radiography results, the patient was a candidate of tonsillectomy and was known as an indexed serous acute otitis media. The paraclinic examinations (including tympanometry and audiometry) were done blindly by one person. And results of innovative treatment method indicate that all of the signs and symptoms were resolved after 1 week of starting treatment. Recurrence of ear infections was followed for 1 year.

\subsection{Audiometric Results After 5- Episodes of Empirical Treatments}

\subsubsection{Audiometry}

Pure Tone Audiometry (PTA) indicate a mild transitional conductive hearing loss in both ears. The average hearing loss was about $15 \mathrm{~dB}$, and $10 \mathrm{~dB}$ in the right and left ears, respectively. Hearing loss of patient eras was a consequence of serous chronic otitis media. The inner parts (nervous) of both ears have normal performances, and bone conduction of both ears was normal. Speech reception threshold (SRT) frequencies were in accordance with PTA.

\subsubsection{Tympanometry (impedance)}

Tympanometry indicate an abnormal function of middle section of both ears. Compliance was lower than normal in both ears. Middle ear pressure in right and left ears was -140 and -15 , respectively (Figure-1). Tympanogram of right ear was type $\mathrm{C}$, and the left ear was type A tending to type C, indicating an inflammation in both ears with severity in right ear. Prescription of antibiotics, urgent referring to physician in viral flues, and personal health were recommended to patient by ENTs and audiometrist to prevent exacerbations of AOM recurrences. In addition, irrigation of nasal cavity with normal saline was also recommended for patient.

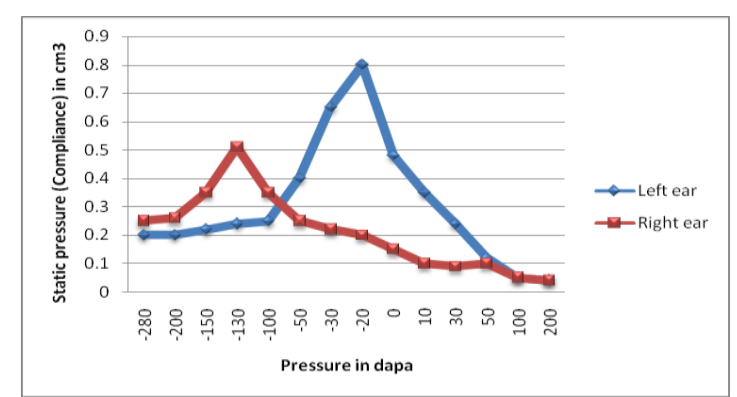

Fig1. Tympanogram after 5 treatment regimens which have been failed finally, as indicated by negative pressure in both of the ears 


\subsection{Audiometric Results after 6th Episode of New Regimen (1 Week after Starting Treatment):}

After taking amoxicillin, regular washing of the nose with saline solution, and nanosilver and ethanol solutions recommended for treatment, and test results showed very fast and good recovery. Both ears showed a normal PTA and SRT. SRT of both ears was completely normal (3-5 db). Internal pressure of right and left ears was reported zero and +15 by tympanometry, respectively. This in turn refers to lowering of negative pressure in ears and recovery of middle ear inflammation. Compliance of both sides was acceptable, and movement of tympan and middle ear ossicles was normal. In otoscopy examination there wasn't seen tympan inflammation and abnormalities in middle ear bones or eardrum bulgings. Also discharge in nose, ear, and throat was not observable (pyogenic sputum).

Tympanogram of patient after successful treatment has been indicated in figure-2. After 1 year of follow up there was not seen inflammation or complications, and side effects in patient.

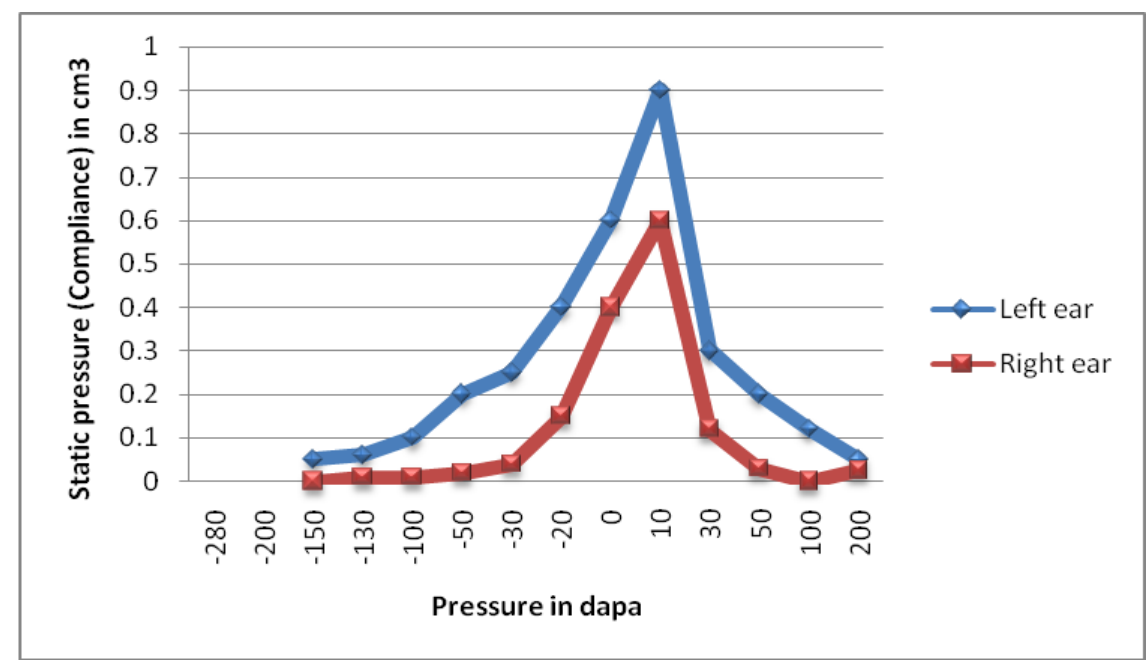

Fig2. Tympanogram after $6^{\text {th }}$ treatment regimen, the red and blue graphs indicating internal pressures of the right $(+10 \mathrm{db})$ and left $(+10 \mathrm{db})$ ears, respectivel

\section{DISCUSSION}

Results of this study shows that treatment with high dose antibiotics and nanosilver a known broadspectrum antimicrobial, and external interventions including regular irrigation with normal saline and ethanol can be successful in treatment of recurrent AOM, and very reliable. Results are very excellent because after 1 year follow up there was not seen ear related complaint of patient. The treatment method has eliminated all of the recurrent signs and symptoms of AOM, and audiometric parameters have reversed to normal levels. Recurrence of AOM attacks in lowers than 1 month after treatments shows a typical failure of therapy, which in turn indicates presence of a mixed bacterial etiologies or viral synergisms. In some studies indicated that AOM failures are higher than other infections, and in about $10 \%$ of cases treatment failures have been occurred against first choice antibiotics(5). In a study, rate of failures when originally treated with amoxicillin have been estimated to more than $60 \%$ (6). Although this study may have problems from viewpoint of medical research ethics, but considering the condition of patient and discomfort in a 2 year course, and after getting agreements of parents and child, the treatment method applied. It should be mentioned that all the materials used are already used by humans, which have no distinguished side effects. Moreover, patient was detected as an urgent selective tonsillectomy by 3 different ENTs So that the risk of hearing loss, and other complications was anticipated for the patient.

\section{CONCLUSION}

This method has recommended as a successful treatment for resistant and recurrent forms of AOM, and it has been proposed to be considered in further studies of clinical trials.

Nanosilver particles have persistent antimicrobial effects in vivo, so limited and topical applications of diluted concentrations could have synergism with other antimicrobials in combating with infections without side effects. In addition external intervention by approved broad spectrum antimicrobials including nanoparticles could have acceptable effects in treatment resistant cases such as AOM. 


\section{REFERENCES}

[1] Zielnik-Jurkiewicz B, Bielicka A. Antibiotic resistance of Streptococcus pneumoniae in children with acute otitis media treatment failure. Int J Pediatr Otorrhinolaringol 2015;30:S0165-5876(15)00477-2

[2] Mark SG. Treatment failure, recurrence, and antibiotic prescription rates for different acute otitis media treatment methods. Clin Pediatr 2010; 49(10): 970-75.

[3] Michael EP, Adriano A, Ron D, et al. Safety and efficacy of Gatifloxacin therapy for children with recurrent acute otitis media (AOM) and/or AOM treatment fFailure. Clinic Infect Dis 2005; 41:470-78.

[4] Kozyrskyj AL, Klassen TP, Moffatt M, Harvey K. Short course antibiotics for healthy children with uncomplicated acute otitis media. Cochrane Library 2010;

[5] Craig J C, Ellen B, Sara JJ, Chris DP, Mario O, Stefan D, et al. Antibiotic treatment failure in four common infections in UK primary care 1991-2012: longitudinal analysis. BMJ 2014; 349:g5493.

[6] Natália B, Roger A M J, Arno WH, et al. Recurrence up to 3.5 years after antibiotic treatment of acute otitis media in very young Dutch children: survey of trial participants. BMJ 2009; 338

Citation: H. Hosainzadegan, "Application of Combination of High-Dose Amoxicillin with Nano-Silver and 70\% Ethanol for the Treatment of a Recurrent Middle Ear Infection: A Case Report", ARC Journal of Pharmaceutical Sciences (AJPS) , vol. 3, no. 3, p. 9-12, 2017. http://dx.doi.org/10.20431/2455-1538.0303003

Copyright: (C) 2017 Authors. This is an open-access article distributed under the terms of the Creative Commons Attribution License, which permits unrestricted use, distribution, and reproduction in any medium, provided the original author and source are credited. 